\title{
Morphological organization of the fimbria and fornix of hippocampus in the fox
}

\author{
Iwona Łuszczewska-Sierakowska ${ }_{\mathrm{A}, \mathrm{B}, \mathrm{D}, \mathrm{E}, \mathrm{F}, \mathrm{G}}$, Małgorzata Matysek ${ }_{\mathrm{B}, \mathrm{G}}^{1}$, Agata Wawrzyniak-Gacek ${ }_{\mathrm{E}, \mathrm{P}}^{1}$ \\ Anna Charuta $_{\text {B, D }}^{2}$, Krzysztof Chmielowiec \\ ${ }^{1}$ Chair and Animal Anatomy and Histology, Faculty of Veterinary Medicine, University of Life \\ Science in Lublin \\ ${ }^{2}$ Chair of Zoology, Vertebrate Morphology Laboratory, University of Life Science in Siedlce \\ ${ }^{3}$ Chair of Animal Physiology
}

\begin{abstract}
The hippocampus is one of the essential brain structures in humans and animals. It belongs to the limbic system and plays an important role in long-term memory and spatial orientation. Many structures of the limbic system of the prosencephalon connect with the hypothalamus through the C-shaped structures (such as hippocampus and fornix) and with the amygdale and terminal stria. The fimbria and fornix of hippocampus form a complex system of afferent and efferent nerve fibres closely related functionally and structurally with the hippocampal formation and other brain parts. The fimbria of hippocampus, which is a broader band of nerve fibres, spreads over the anterior-medial surface of the hippocampal body. The fimbria and crus have been studied in pigs, rabbits, opossums and monkeys. In terms of topography, cytoarhitectonics and morphology, the nerve fibre structures found in foxes/minks are similar to those described in other domestic animals, including the fornix, which is an arched-shaped aggregation of fibres between the hippocampus and mammillary body and the fimbria. The present study was conducted in 5 adult foxes using the Klüver and Barrery method as well as the Landau method modified according to Mirando. Morphological analysis was performed and pictures were taken in the Cell $\wedge$ D Soft Imaging System (SIS) combined with a digital camera Colorview IIIu.
\end{abstract}

Keywords: hippocampus, fimbria, fornix

\section{Introduction}

The hippocampus is one of the essential brain structures in humans and mammals. It is part of the limbic system and plays an important role in long-term memory and spatial orientation. Many structures of the limbic system of prosencephalon connect with the hypothalamus via the C-shaped structures (such as hippocampus and fornix) and with the amygdale and terminal stria. The hippocampal formation and its tracts in the fornix bend towards the anterior pole of the diencephalon forming the precommissural (septal) and postcommissural (hypothalamic) connections in relation to the anterior commissure. The fimbria of and fornix constitute a complex system of nerve fibres connecting the hippocampus with other brain structures $[1,2,3]$. According to recent experimental studies carried out in various species, the fornix is a pathway for afferent and efferent nerve fibres closely connected with the hippocampal formation. In their study in rats, Meinbach et al. [4] determined the accurate localization of efferent nerve fibres in the fimbria and fornix of hippocampus. There are numerous literature data regarding the morphology and topography of the fimbria and fornix in rats, mice, monkeys, minks and opossums $[5,6,7,8,9,10,11]$ and no studies in other species, particularly fur-covered animals, such as foxes, which is the aim of the present study.

\section{Material and methods}

The study was conducted in 5 adult foxes. Cerebral crania were opened, brains collected and fragments containing the hippocampus prepared. The Klüver and Barrery method and the Landau method modified according to Mirando were used. The hippocampus was fixed in formalin, dehydrated in ethyl alcohol and embedded in paraffin blocks. The $10 \mu \mathrm{m}$ paraffin sections were cut using a microtome in the frontal and horizontal plane. The preparations were stained with the luxol fast blue and cresyl violet. Morphological analysis was performed and pictures were taken in the Cell $\wedge$ D Soft Imaging System (SIS) combined with a digital camera Colorview IIIu.

\section{Results and discussion}

The fimbria and fornix of hippocampus form a complex system of afferent and efferent nerve fibres closely related functionally and structurally to the hippocampal formation and other brain parts (fig.1). The fimbria of hippocampus, as a broader band of nerve 
fibres, spreads over the anterior-medial surface of the hippocampal body (fig.3). In the sagittal cross-section, the ventral layer of nerve fibres of the fimbria forms a marked eminence near the fornix crus (fig.2), separated from the hippocampal dentate gurus by the fimbriodentate sulcus. In transverse sections, the fimbria forms a broad band of nerve fibres connected with the dorsal surface (stratum oriens) of ventral hippocampus and its ventral surface of the dorsal part. The dorsomedial part of fimbria prolongs into the fornix crus. In rats, the fornix consists of the onis fornicis, columna fornicis, comnissura fornicis dorsalis and comnissura fornicis ventralis. The band of nerve fibres that forms the fornix crus is dorsally-ventrally flattened near the fimbria to become cylindrical medially. At the level of the posterior border of the fornix dorsal commissure, the fornix crus prolongs itself into the fornix body. The fibres of fornix crus are relatively thick and well myelinated. The fornix body is the central part of fornix; its posterior segment divides into the right and left parts, which prolong into the fornix crus. The fornix commissure, also called psalterium ventrale, is formed by transversally placed nerve fibres that connect the right and left beginning of fornix body. The ventral commissure of fornix, also termed psalterium ventrale, is located in the posterior septal area. It is composed of transversally and obliquely placed nerve fibres connecting the descending ventral columns of fornix.

The hippocampal fimbria and crus have been studied in rats, opossums and monkeys $[9,10,12]$. The nerve fibre structures described in foxes and minks are similar to those found in other animal species in terms of topography, cytoarhitectonics and morphology.

The hippocampus is a complex system of afferent and efferent nerve fibres connecting the individual structures of the limbic system and its structures with brain parts. Morphologically, the following hippocampal parts can be distinguished: the alveus, fimbria and fornix. The fornix is a nerve tract composed of afferent and efferent nerve fibres of the hippocampus. The study findings in monkeys suggest that afferent nerve fibres are found in the ventral fornix [8]. The afferent nerve fibres in the fornix originate from the septal area structures [10]. More detailed studies concerning topographic localization of afferent nerve fibres of the individual hippocampal structures have demonstrated that afferent nerve fibres in the fimbria and fornix head towards their destination [10]. The efferent connections of hippocampal formation together with the septal area nuclei, pre-visual area, dorsal hypothalamic field, anterior thalamic area and mammillary region pass through the fornix [13]. Unlike the afferent nerve fibres, the efferent ones pass through the dorsal part of hippocampal fornix and fimbria [13,14]. The system was morphologically well described in various species many years ago, e.g. in Guinea pigs, monkeys $[8,10]$. In foxes, the structures in question are morphologically and topographically extremely similar to those described in other domestic animals, both the fornix, which is an arched-shaped aggregation of fibres between the hippocampus and mammary body and the fimbria. The fimbria of hippocampus originates from the hippocampus and passing the inferior horn of the lateral ventricle prolongs itself into the fornix crus. The crus of both hemispheres unites forming the fornix at the level of the corpus callosum. The nerve fibres building the fornix body run in the ventral direction dividing into the right and left parts as columns of fornix. The recent methods of nerve fibre mapping which employ autoradiography provide more comprehensive and detailed data on the location, course and topography of nerve fibres in the fimbria, fornix and individual hippocampal areas [4, 15]. Using the above methods, Meinbach et al. described topographic localization of efferent nerve fibres of individual structures of the hippocampal formation, fimbria and fornix in the rat. They divided the structures (fimbria and fornix) composed of nerve fibres into 5 zones. The zone 1 involves the medial part of fornix plus ventral part of fimbria and contains axons running from the anterior-dorsal part of hippocampus whereas the zone 2 is formed by the lateral part of fornix and the adjacent ventral part of fimbria. The zones 3 and 4 contain nerve fibres in the medial region of fimbria and are placed laterally to the fornix. The zone 5 refers to the terminal lateral part of fimbria and is composed of axons of the anterior-ventral part of hippocampal formation. The authors analysed more accurately the topographic localization of fibres considering the individual hippocampal structures (regio interior, regio superior, subiculum). Moreover, their findings reveal that the subiculum cortex is the only source of fornix fibres, which run to the hypothalamus; its lateral part contains efferent nerve fibres running to the mammillary body nuclei. The study by Wyss et al. [15], in which autoradiography was used, regarded injections of $3 \mathrm{H}$-amino acids into the relevant hippocampal areas. The newest techniques are required to localize the nerve fibre and determine its course in such a precise way. 


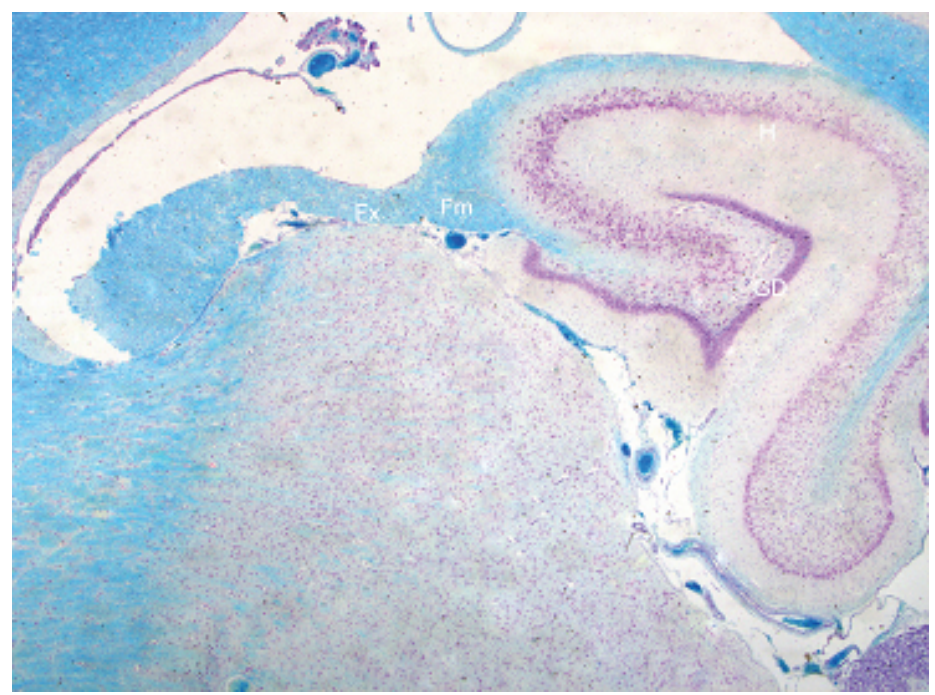

Fig. 1. Transverse cross-section of the cerebral hemisphere near the medial plane according to Klüver and Barrery. $125 \mathrm{x}$ magnification

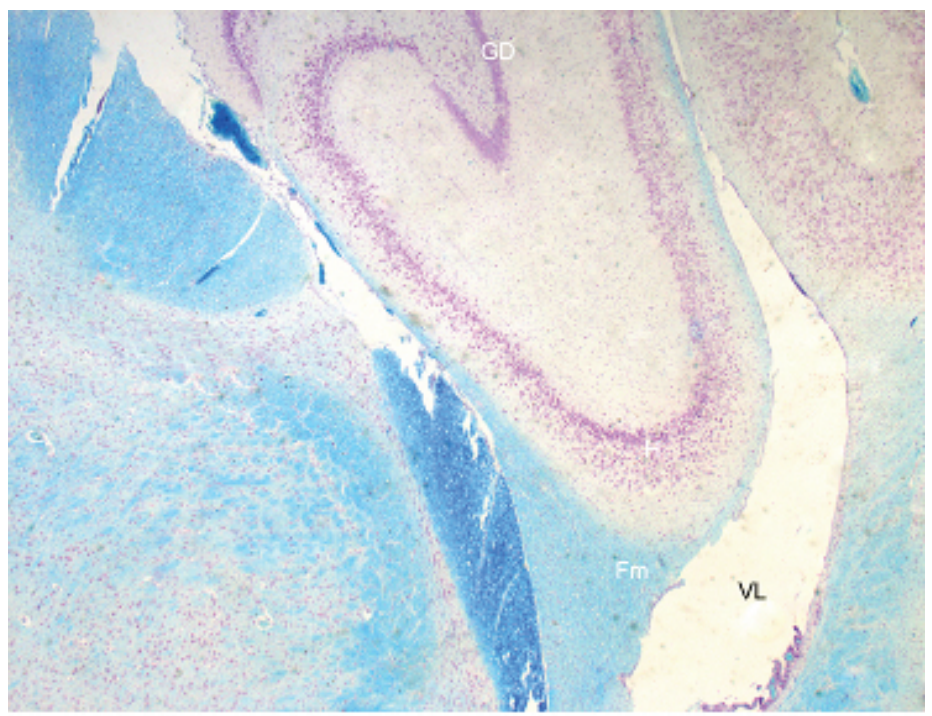

Fig.2. Transverse cross-section of hippocampus formation according to Klüver and Barrery. 125x magnification

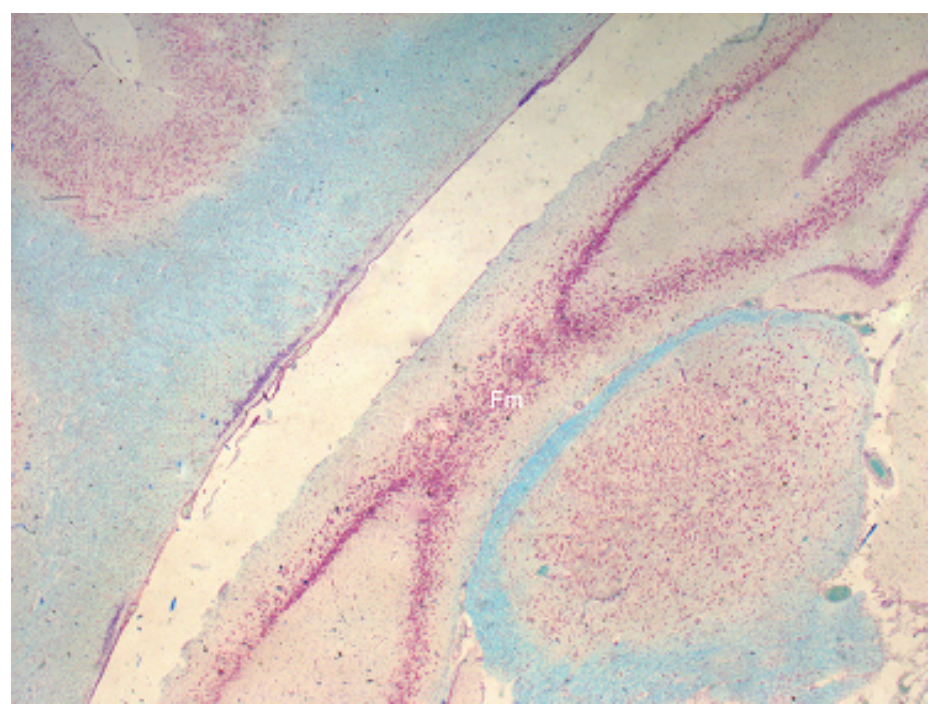

Fig.3. Sagittal cross-section of hippocampus formation (dorsal and ventral) according to Klüver and Barrery. 125x magnification 


\section{References}

1. Aguilar J.S., Jerusalinsky D., Stockert M., Medina J.H., De Robertis E. Localization of hippocampal muscarinic receptors after kainic acid lesion of CA 3 and fimbria-fornix transection. Brain Res., 1982; 16; 247(2) :335-40.

2. Halasy K., Hajszan T., Kovács E.G., Lam T.T., Leranth C. Distribution and origin of vesicular glutamate transporter 2-immunoreactive fibers in the rat hippocampus. Hippocampus, 2004; 14(7): 908-18.

3. Daitz H.M., Powell T.P.S. Studies of the connections of the fornix system. J. Neurol. Neurosurg. Psychiat, $1954 ; 17,75$.

4. Meinbach R.C., Siegel A. Efferent connections of the hippocampal formation in the rat. Brain Res., 1977; 124, 197. 5. Grin'kina N.M., Abdel-Baki S.G.,Bernold P.J. Reversible Behavioral Deficits in Rats during a Cycle of Demyelination-Remyelination of the Fimbria. Plos, January 22. 2013;DOI10.1371/journal.

6. Jeserich M.W. The nuclear pattern and fiber connections of certain non- cortical areas of the telencephalon of the mink (Mustela Vision). J. Comp. Neurol., 1945; 83, 173.

7. Katalin H., Tibor H., E'va G. Kova, Thien-Tri L. Csaba L. Distribution and Origin of Vesicular Glutamate Transporter 2- Immunoreactive Fibers in the Rat Hippocampus. Hippocampus, 2004; 14:908-918.

8. Lauer E.W. The nuclear pattern and fiber connections of certain basal telencephalic centers in the macaque. J. Comp. Neurol., 1945; 82, 25.

9. Loo Y.T. The forebrain of the opossum, Didelphis virginiana. J. Comp. Neurol., 1931; 52, 51.

10. Mc Lardy T. Observations on the fornix of the Monkey. I. Cell studies. Ibid, 1955; 103, 305.

11. Shinji T. Osamu M., Najma A. Janjua C., Tetsuji M.,Fumio T., Ryoji K., Toshifumi I. Stage and region dependent expression of a radial glial marker in commissural fibers in kindled mice. Epilepsy Research, 2005; 67: 61-72.

12. Nauta W.J.H. An experimental study of the fornix system in the rat. J. Comp. Neurol., 1956; 104, 247.

13. Powell E.W. Septal efferents revealed by axonal degeneration in the rat. Exp. Neurol., 1963; 8, 401.

14. Humprey T. The telencephalon of the bat. I. The non-cortical nuclear masses and certain pertinent fiber connections. J. Com. Neurol., 1936; 65, 603

15. Wyss J.M., Swanson L.W., Cown W.M. The organization of the fimbria, dorsal fornix and central hippocampal commissure in the rat. Anatomy and Embryology, 1980; 158(3): 303-316.

Corresponding author's address:

Iwona Łuszczewska-Sierakowska

Uniwersytet Przyrodniczy w Lublinie

Katedra Anatomii i Histologii Zwierząt

ul. Akademicka 12

20-950 Lublin

iwona.luszczewska@up.lublin.pl 